L'HOMME L'Homme

$217 \mid 2016$

Varia

\title{
Structures des groupes humains
}

Vers une axiomatique

Structural Properties of Human Organizations. An Essay in Axiomatics

\section{Charles Macdonald}

\section{CpenEdition}

Journals

Édition électronique

URL : http://journals.openedition.org/lhomme/28814

DOI : 10.4000//homme.28814

ISSN : 1953-8103

Éditeur

Éditions de l'EHESS

Édition imprimée

Date de publication : 25 février 2016

Pagination : 7-20

ISSN : 0439-4216

Référence électronique

Charles Macdonald, «Structures des groupes humains », L'Homme [En ligne], 217 | 2016, mis en ligne le 24 février 2018, consulté le 10 décembre 2020. URL : http://journals.openedition.org//homme/ 28814 ; DOI : https://doi.org/10.4000//homme.28814

(c) École des hautes études en sciences sociales 


\title{
Structures des groupes humains
}

Vers une axiomatique

\author{
Charles Macdonald
}

$\mathrm{L}_{\mathrm{A}}$

A DOUBLE NATURE du social a été largement reconnue par maints auteurs en termes de "grand partage " et en fonction d'un certain nombre de dichotomies (primitif/civilisé, traditionnel/moderne, politique/social, etc.) (Fox 2011 ; Macdonald 2011a). Sans devoir remonter à Rousseau, quelques sociologues comme Ferdinand Tönnies (1955), des philosophes comme Martin Buber (1992) ${ }^{1}$, ou des ethnologues comme Pierre Clastres (1974), Victor Turner (1974), ou encore Stanley Diamond (1974), ont bien deviné la présence de principes antithétiques et compris qu'il existait deux manifestations radicalement différentes de la vie collective. Plus récemment encore, des sociologues ont mis au jour deux structures, et deux seulement, caractérisant tous les groupes humains : la hiérarchie et la coopération (Van Meter 2014). Ces approches peuvent-elles aboutir à fonder une théorie de la société ? Je pense que oui, mais à deux conditions. La première est de prendre en considération certains groupes humains observés par les ethnologues, la seconde est d'adopter une perspective évolutionnaire. Ces deux conditions permettent de situer le schéma dans une problématique anthropologique large et amènent à redéfinir les

1. Sur le «double principe de la vie humaine», selon Martin Buber, cf. Buber (1992: 61 et passim) et sur le social et le politique (Ibid.: 205 sqq).

Cet article reprend des hypothèses développées dans une série de publications antérieures (Macdonald 2008a et b; 2009; 2011a, b et c, 2012a et b; 2013;2014a et b), et sur deux sites internet personnels (https://sites.google.com/site/charlesjhmacdonaldssite/; https://anarchogregaire.wordpress.com/), pour en donner une vue synthétique fondée sur une argumentation recentrée sur la coopération et l'autonomie. Une version préliminaire de ce texte est accessible en ligne sur la plateforme Hypothèses [http ://aggiornamento.hypotheses.org/2380]. Je remercie Joël Candau pour ses remarques. 
concepts fondateurs. J'utiliserai ainsi, dans un sens précis et particulier, celui de régime "socio-hiérarchique" et je lui opposerai celui de régime " anarcho-grégaire» ou "anarcho-égalitaire " ${ }^{2}$. J'opposerai de la même façon la réciprocité au partage ${ }^{3}$, les liens faibles aux liens forts, dans un rapport homothétique au couple égalité/hiérarchie.

\section{Axiomatique}

On posera en premier lieu que toute vie collective humaine est fondée sur la coopération et l'implique nécessairement ${ }^{4}$. La coopération est l'activité coordonnée de sujets multiples pour obtenir un bénéfice commun (trouver de la nourriture, construire un pont, chanter en chœur, reproduire l'espèce, élever des enfants). Si, par ailleurs, les sujets individuels de la vie collective sont, comme dans le cas des Homo sapiens, des sujets autonomes (dont le comportement relève de lois internes au sujet) ${ }^{5}$, alors le problème central de toutes les sciences sociales est celui de la compatibilité entre l'autonomie du sujet et la nécessité de la coopération.

Il est possible de rendre le sujet moins autonome, de limiter son autonomie, voire même de le rendre hétéronome, comme c'est le cas dans la grande majorité des sociétés post-néolithiques. Cette situation relève de facteurs internes et externes au sujet qui, ontologiquement, reste autonome dans son être. Il est également possible de conserver l'autonomie du sujet sans la limiter. C'est encore le cas dans de rares groupes humains qui vivent

2. J'utilise, faute de mieux, ce terme composite. La composante anarchique ("anarcho-») a deux sens : 1) qui n'a pas de chef, sans hiérarchie ; 2) qui est aléatoire, non linéaire. La composante "grégaire " se réfere uniquement à la propriété de vivre en troupeau ou en groupe, par opposition aux espèces animales qui vivent à l'état solitaire. Le terme « anarchogrégaire " signifie donc « qui vit dans des collectifs sans principe d'ordre hiérarchique». On peut lui préférer le terme "anarchoégalitaire ", ou utiliser l'expression de "collectifs faiblement agrégés " proche de l'anglais openaggregated (Gibson \& Sillander 2011).

3. Comme il sera précisé plus loin, le partage est une forme de distribution sans dette. Le partage s'oppose à la réciprocité, ou l'échange, dans la mesure où le schéma don/contre-don ne s'applique pas.

4. La notion de coopération a dominé les discussions conduites par les biologistes et les théoriciens de l'évolution (cf. West, Griffin \& Gardner 2007; Candau 2012). Cette notion est liée à celles de succès reproductif, d'altruisme et de gène "égoïste » et associées dans les théories de sociobiologie et de sociologie évolutionnaire, aux noms de Dawkins, Hamilton, Trivers, Wilson, entre autres. La coopération est un fait très largement biologique et concerne beaucoup d'espèces animales, mais la coopération humaine pose des problèmes spécifiques de rationalité. Les problématiques fondées sur le "dilemme du prisonnier" d'Albert Tucker et sur la "tragédie des terres communales" de Garet Hardin ont constitué une tentative pour résoudre le problème de la spécificité et de la rationalité de la coopération humaine (Ostrom 1990). Il est possible que les formes primitives de coopération humaine aient concerné prioritairement la protection et le soin des nouveaux-nés, ainsi que des activités collectives ludiques (musique, danse).

5. L'autonomie du sujet est le principe premier qui conditionne l'égalitarisme (Boehm 1993). La notion d'autonomie individuelle est directement liée à celle de lien faible, envisagée plus bas. 
à l'état nomade ou semi-nomade ${ }^{6}$. La thèse la plus vraisemblable est que cette condition a été celle de l'Homo sapiens jusqu'au paléolithique supérieur ${ }^{7}$.

On peut alors poser les axiomes ou postulats suivants :

\section{A - Sujets autonomes et coopération = égalité entre les sujets \\ $\mathrm{B}-$ Sujets non autonomes et coopération $=$ hiérarchie $^{8}$}

En effet, ou bien les sujets sont autonomes et la coopération est volontaire, ce qui entraîne une équivalence entre les sujets de la coopération; ou bien les sujets ne sont pas autonomes, la coopération est forcée et les sujets ne sont pas équivalents dans la coopération et eu égard au résultat de la coopération.

L'équivalence ou la non-équivalence des sujets a des conséquences importantes dans tous les domaines de l'activité humaine, particulièrement sur les plans économique, politique et moral. Ces deux formules opposées ( $\mathrm{A}$ et $\mathrm{B})$ produisent deux modalités différentes de vie collective. La modalité $A$ est à la fois aléatoire, interactive et réticulaire; la modalité $B$ est linéaire, ordonnée et fonctionne sur la base de groupes disjoints. J'appelle les types de collectifs du groupe $\mathrm{A}$ « anarcho-égalitaires » ou

6. Parmi les cas typiques citons ceux, classiques, des chasseurs-cueilleurs hadza (Woodburn 1979, 1982), mbuti (Turnbull 1968), !kung (Thomas 1989 [1958]), inuit (très nombreuses publications d'auteurs, parmi lesquels les noms de Birket-Smith, Boas, Rasmussen, Damas, Guemple, Robbe, Freuchen, Malaurie doivent être mentionnés), paliyan (Gardner 2006 : 30 sqq.). Des essarteurs (agriculteurs) appartiennent également à cette catégorie, notamment les Buid (Gibson 1986), les Semai orientaux (Dentan 1968), les Palawan (Macdonald 1977, 2007), les Majangir (Stauder 1972). En font aussi partie des nomades marins comme les Bajau Laut (Sather 1997). La liste peut être considérablement allongée.

7. L'argument généralement avancé est que Homo sapiens vit jusqu’au paléolithique supérieur dans la condition de chasseur, cueilleur, charognard et que les petits groupes de chasseurs-cueilleurs modernes, pédestres (à ne pas confondre avec les chasseurs équestres ou les sociétés de pêcheurs sédentaires) appartiennent tous à la même classe de sociétés dites "à bandes ", fondées sur les principes de la mobilité, de la fission-fusion et de l'égalitarisme, ou comme le veut Tim Ingold (1999) et comme je le défends moi-même, sur ceux de l'immédiateté, de l'autonomie et du partage (et en conséquence ne relevant pas de la définition habituelle d'une organisation sociale). Malgré les changements survenus chez les chasseurs-cueilleurs des XIXe et XXe siècles, et en dépit de la présence de grandes sociétés agraires ou industrielles et des interactions avec elles - ce qui a pu faire même douter de l'authenticité des premiers -, il reste vraisemblable que les principes de l'organisation des chasseurs-cueilleurs avant le néolithique ressemblaient à leurs homologues contemporains. Dans un ouvrage remarquable, l'ethnomusicologue Victor Grauer démontre, à partir d'une analyse musicologique et culturelle, et d'une classification des haplogroupes définis sur la base de l'ADN mitochondrial que les groupes humains qui sont passés d'Afrique en Asie, il y a environ 70000 ans, étaient étroitement apparentés aux groupes de Pygmées africains contemporains, comme les Ju/'Hoansi, !Kung, Aka, Baka et Mbuti, et qu'ils relevaient d'une origine commune. Or, tous ces groupes présentent les mêmes traits d'organisation mentionnés ci-dessus (Grauer 2015 [2011]).

8. Je distingue la hiérarchie qui suppose le pouvoir, de l'asymétrie simple qui repose sur l'autorité morale ou rationnelle et qui n'est pas transitive. La hiérarchie est une relation d'ordre partiel (Sade 1992). Dans un article important, Alan Page Fiske (1992) distingue quatre sortes de .../... 
"anarchogrégaires " 9 , les types du groupe B «socio-hiérarchiques». La quasi-totalité des populations humaines actuelles appartient au groupe B (les organisations sociales ou socio-hiérarchiques). Quelques populations humaines encore vivantes et la totalité de la population humaine jusqu'au paléolithique supérieur ou récent existent et ont existé selon la modalité A, anarcho-égalitaire ou anarchogrégaire ${ }^{10}$.

Toute forme de vie collective humaine implique, ai-je dit, la coopération. Ce qui differe radicalement dans les deux modalités $A$ et $B$, c'est le degré d'autonomie du sujet. Dans la première, les individus sont et restent égaux entre eux. Il n'y a pas d'ordre dans les deux sens du mot. Il n'y a pas de corporations, mais des réseaux. Enfin, la répartition des biens et des ressources repose sur le principe du partage entre les membres de la communauté sans que personne ne donne à personne (il n'y a pas de dette) ${ }^{11}$.

Dans la modalité $\mathrm{B}$, «socio-hiérarchique », le principe hiérarchique (ordre partiel) détermine l'ensemble de l'organisation. Il permet l'émergence de corporations (par exemple sur la base de la filiation, comme le clan, ou sur

[Suite de la note 8] relations ou formes de socialité élémentaires: le partage communautaire (communal sharing, CS), la hiérarchie (authority ranking, AR), l'égalitarisme (equality matching, $\mathrm{EM}$ ) et les valeurs du marché (market pricing, MP). La première (CS) est une relation d'équivalence (réflexive, symétrique et transitive), la deuxième (AR) est un ordre linéaire (réflexif, transitif et antisymétrique), la troisième (EM) a les propriétés d'un groupe abélien, commutatif, avec la possibilité d'addition et de soustraction. Le dernier (MP) repose sur le principe de proportionnalité qui suppose les propriétés de division et de multiplication. Lidée que les relations humaines et les formes de vie collective puisset être ramenées à quelques relations élémentaires formalisables en langage mathématique est certainement une idée scientifiquement intéressante. Dans la formulation que j’ai adoptée $: C S$ = partage, $\mathrm{AR}$ = hiérarchie, $\mathrm{EM}$ = réciprocité. Je n'envisage pas le quatrième terme (MP) qui s'applique prioritairement aux organisations sociales complexes fondées sur les valeurs du marché et qui définit aussi un jugement sur l'humain (en rapportant la valeur d'un individu à sa richesse pécuniaire, par exemple). On voit bien que la seule relation qui suppose que tous les éléments de l'ensemble sont équivalents est celle du partage (CS), les deux autres sont définies comme des relations d'ordre dont tous les éléments ne sont pas équivalents. L'égalité se définit par le partage.

9. Cf. supra note 2.

10. Cf. : notes 6 et 7 ci-dessus, et aussi Christopher Boehm (199: chap. IX).

11. De nombreuses discussions, analyses et interprétations ont été proposées pour classer et expliquer les multiples formes de transaction, distribution et circulation des produits dans les sociétés humaines et animales (cf., par exemple, sur les différentes modalités du partage: Wenzel, Hovelsrud-Broda \& Kishigami 2000). C'est à James Woodburn que nous devons le constat définitif que le partage est une forme de transaction conceptuellement et empiriquement distincte de la réciprocité (Woodburn 1998). Celle-ci pose, dans le modèle maussien classique (Mauss 1925), un don et un contre-don et la triple obligation de donner, de recevoir et de rendre. Elle implique, au premier chef, la dette, soit une relation asymétrique entre donneur et preneur, relation qui ouvre la voie à la subordination du preneur (le débiteur). Le partage quant à lui exclut la dette. Dans la pratique, le partage et la réciprocité interviennent successivement ou alternativement, avec des formes intermédiaires comme le don pur (confondu avec le partage), la redistribution, le vol toléré, ou le don forcé (Peterson 1993). La réciprocité existe partout mais le point important est que le partage fournit le modèle d'une éthique relationnelle, qui est prépondérante dans les systèmes anarchoégalitaires. Pour une discussion et une bibliographie plus complètes, cf. aussi Macdonald (2008b). 
la base d'un être collectif transcendant, comme le royaume ou l'État). Enfin, il suppose la répartition des biens et des ressources sur la base de la réciprocité qui elle-même suppose la dette qui lui donne vie. La dette, en effet, instaure de fait une relation asymétrique. Elle implique la supériorité du donneur sur le preneur et induit une hiérarchie potentielle.

\section{Liens forts et liens faibles ${ }^{12}$}

Pour exister dans la modalité A (anarcho-égalitaire), les sujets autonomes doivent former entre eux des liens négociables. Ces négociations aboutissent à renforcer ou, au contraire, à abolir les liens existant pour les remplacer par d'autres, de façon constante. Les réseaux sont sans cesse renouvelés, ils sont aléatoires et soumis à l'entropie ${ }^{13}$. Les liens sont faibles. Dans la modalité B (socio-hiérarchique), en revanche, les liens sont forts ou pensés comme tels et dépendent d'un tiers, lui-même transcendant. Les ensembles constitués par les sujets sont indépendants de la volonté des sujets (qui ne sont plus complètement autonomes de ce fait). Ils sont fixes et résistent à l'entropie. Le modèle d'organisation tend à être linéaire et mécanique ${ }^{14}$.

12. La notion de « lien faible » est empruntée à Mark Granovetter (1973), mais avec les conséquences évolutionnaires mises à jour par Alexandra Maryanski (1994), et son collègue Jonathan Turner (Maryanski \& Turner 1992). On pourra également consulter à ce sujet Charles Macdonald (2008a, 2009, 2011a et b, 2012a). Par lien faible, j'entends ici des liens interpersonnels qui sont soumis à la volonté des partenaires ainsi liés. Ces liens peuvent être créés ou abolis sans recours à une autorité tierce. Ils sont immanents. Les liens forts sont des liens permanents soumis à une autorité tierce. Ils sont typiquement des liens institutionnels et tendent à être pensés comme transcendants. L'amour érotique est typiquement un lien faible (qui se dissout en fonction du désir des partenaires), le mariage est un lien fort (qui ne peut être contracté ou dissous qu'avec l'accord de l'Église ou de l'État, et qui est ou était pensé comme sacré).

13. L'entropie qui est considérée dans ces pages concerne essentiellement la composition des groupes ou collectifs dont l'ordre initial est sans cesse transformé. Dans la modalité anarchoégalitaire, les collectifs faiblement agrégés se renouvellent constamment et changent de composition. Ils possèdent et conservent cependant une identité durable. Il en va de même pour les cellules du corps humain qui meurent et sont constamment remplacées par de nouvelles. L'ensemble du corps conserve son unité mais ses éléments constitutifs changent à travers le temps. Les collectifs du type anarchogrégaire obéissent ainsi à une loi organique qui veut que le tout soit plus durable que les parties (en opposition à une loi mécanique qui fait que les parties sont plus durables que le tout). Edgar Morin a admirablement développé cette idée empruntée au mathématicien et pionnier de la cybernétique, John Van Neuman, en l'appliquant aux organisations complexes (Morin 2005 : 43-44). Corollairement, la prévisibilité de l'ordre collectif purement grégaire est faible.

14. La solidarité organique, selon Émile Durkheim, est en fait une solidarité de type strictement mécanique, fondée sur l'analogie avec l'anatomie du corps humain et supposant la simple complémentarité entre les différents organes (cour, foie, tête, etc.). Dans ce modèle si admirablement conforme à un idéal hiérarchique, la tête représente évidemment la classe supérieure dans la société (particulièrement les brahmanes et les sociologues). Le modèle anarcho-égalitaire suppose que la tête est partout. Cf., à ce sujet, Ori Brafman \& Rod Beckstrom (2006). Quand la complémentarité mécanique prend la forme d'une classification (structure en arbre), on parle d'holisme ou de totalité holistique. 
La création et le renforcement de liens faibles pour aboutir à une coopération temporaire supposent certaines conditions, dites ici « conditions de félicité » ${ }^{15}$, justiciables de critères inhérents aux sujets autonomes, donc immanentes aux sujets concrets et à leurs conditions. La création et le maintien de liens forts nécessitent de leur côté deux opérations distinctes : la contrainte externe et l'adhésion interne. Celle-ci repose sur le principe de transcendance et de son extension à l'action, ici nommée «transcendantisme ». Dans la modalité $B$, en effet, les sujets restent autonomes mais subordonnent leur autonomie personnelle à un vouloir collectif ${ }^{16}$.

\section{L'ontologie transcendantiste et le principe corporatif}

La modalité B repose sur une ontologie particulière. Celle-ci comporte deux aspects interdépendants. Le premier pose l'existence d'entités abstraites mais réelles, douées d'une supériorité ontologique et morale, et d'une durée intemporelle et permanente ${ }^{17}$. Le second est que ces entités font partie de l'essence du sujet et le commandent de l'intérieur ${ }^{18}$. Le transcendantisme permet ainsi le passage de l'autonomie à l'hétéronomie, en conservant l'illusion ou la croyance en l'autonomie. Cette opération est celle qui fonde le social.

15. L'expression est empruntée à la linguistique (Austin 1962), mais légèrement détournée de son sens premier. Elle signifie ici : conditions qui permettent l'établissement et la répétition de relations interpersonnelles comme, par exemple, des marques de sympathie ou de politesse, par opposition à l'indifférence ou l'hostilité. En régime anarcho-égalitaire, ces conditions sont nécessaires. L'humour, la plaisanterie et le rire font partie du répertoire des marques de relations souhaitables ou possibles et permettent de créer et de renforcer des liens faibles. C'est pourquoi, en régime anarcho-égalitaire, ces conditions de félicité ne sont pas de simples ornements ou traits secondaires de la vie collective, mais ses conditions indispensables. Des ethnologues se sont avisés de ce fait (Overing 2000).

16. C'est la question posée par Étienne de La Boétie dans le Discours de la servitude volontaire et à laquelle il répondit en affirmant que la soumission était aussi bien le fait des soumis : "Ce sont donc les peuples eux-mêmes qui se laissent, ou plutôt qui se font malmener, puisqu'ils en seraient quittes en cessant de servir. C'est le peuple qui s'asservit et qui se coupe la gorge " (1995 [1548] : 12). La servitude est donc volontaire. Son moyen est, comme je tente de le démontrer ci-dessous, la transcendance et le transcendantisme.

17. Je définis comme transcendant «ce qui dépasse un ordre donné, [...] qui est d'une nature radicalement supérieure ". Cette définition du Petit Robert fait appel à un principe qui dépasse et englobe les particularités ou les différences individuelles, qui est donc universel et en même temps éternel. Je n'utilise pas ici le concept kantien de «transcendantal » qui signifie tout autre chose.

18. Il en va ainsi du sens de l'honneur, du dévouement à une grande cause, de la foi religieuse, du sacrifice de soi. L'obligation d'agir en fonction d'un principe ou d'une entité transcendante (Dieu, la Patrie) est intériorisée par le sujet qui obéit ainsi à une structure idéo-pragmatique. Ce phénomène ressortit à un réflexe d'obéissance et il peut être inculqué par l'éducation et l'apprentissage dès l'enfance. Une très belle illustration de ce fait est donnée dans un article de Merav Shohet (2013) concernant le sacrifice et la socialisation linguistique chez l'enfant vietnamien, dans une société de culture confucianiste imprégnée de transcendantisme patriarcal. La dépendance relève également du phénomène dit "syndrome de Stockholm» et la soumission à l'autorité parentale peut être assimilée à une identification à l'oppresseur. Le sens moral ne ressortit pas seulement au transcendantisme, il appartient d'abord à l'immanentisme comme l'ont si bien expliqué Antisthène, Diogène de Sinope, Épictète et... Georges Brassens. 
Le transcendantisme se présente sous des appellations et des déguisements divers et concerne des objets abstraits ou des "personnes morales " : Patrie, Dieu, Souveraineté, Loi, Intérêt supérieur, Bien commun, Devoir, Obéissance, Honneur, État, République, Peuple, Citoyenneté... Le transcendantisme est la forme générale de l'aliénation.

Par contraste, l'ontologie immanentiste de la modalité anarchogrégaire ou anarcho-égalitaire n'existe qu'entre sujets concrets et porte les noms de sympathie, d'empathie, d'amitié, de solidarité, de plaisir d'être ensemble, d'attachement, d'affection, de force collective joyeuse (common glad impulse $)^{19}$ et de leurs contraires: antipathie, haine, malaise, gêne, méfiance, peur, colère, jalousie, envie...

Le transcendantisme est également le moyen de créer des ensembles disjoints, ou groupes sociaux. C'est le principe corporatifif ${ }^{20}$. Une collectivité est pensée comme une entité abstraite douée de volonté (une quasipersonne); elle possède une supériorité morale; elle est permanente. "Corporations never die ${ }^{21}$. Le vocabulaire juridique français appelle cela, très justement, une "personne morale ". Elle a en effet les attributs du sujet humain mais un sujet divinisé en quelque sorte. La corporation repose sur le principe d'ordre partiel ; elle est donc purement hiérarchique, elle a un chef, des possessions et des limites précises (notamment géographiques). Elle peut obéir au paradigme de la segmentarité ou du centralisme. Son existence est indépendante de la présence des sujets concrets (elle est transcendante de ce point de vue). Enfin, elle est ellemême autonome mais les sujets concrets sont, par rapport à elle, hétéronomes et subordonnés; ils servent à maintenir son existence. Ils y sont contraints par la force mais y adhèrent aussi en leur for intérieur. Le patriote n'est pas seulement fusillé s'il trahit sa Patrie, il souhaite aussi mourir pour Elle. Le transcendantisme est le véritable « Ponzi scheme» de toute l'histoire humaine post-néolithique. Il consiste à faire crédit à un capital fictif ${ }^{22}$. La société sous ce régime suppose toujours l'aliénation.

19. Cf. Charlie Keil (2010).

20. Les corporate groups de l'anthropologie fonctionnaliste britannique (Radcliffe-Brown 1951) sont traduits en français par l'expression "personnes morales ", un concept juridique qui s'oppose à celui de "personne physique». La personne morale est une fiction juridique qui suppose la transcendance. C'est ce que David Graeber appelle «l'abstraction transcendante de la forme corporatiste" (2007: 107). La théorie des corporate groups est à la base de la théorie sociologique fonctionnaliste qui influence encore toute une partie de la sociologie et de l'anthropologie sociale. 21. "There was no escheat of the land to the lord on a death, because such a corporation never dies, and the succession is perpetual ", écrit Henry Sumner Maine en 1875, à propos des anciennes communautés familiales européennes et indiennes (Lecture 1: New Materials For the Early History of Institutions, in Sumner Maine 1888 [1875]).

22. L'État, dans la mesure où il représente la souveraineté nationale, est une entité abstraite. Mais l'État, en tant qu'appareil de gouvernement avec sa bureaucratie et ses forces de police, est.../... 


\section{Coexistence des modalités: le paradoxe de la démocratie}

Les deux modalités de vie collective définies jusqu'ici ne sont pas seulement différentes, elles sont antithétiques, comme le sont en cosmologie la matière et l'anti-matière ${ }^{23}$. Mais chacune suppose l'existence de l'autre dans un même univers.

D'un point de vue évolutionnaire, l'option anarcho-égalitaire est la solution primitive, celle qui a formé les émotions, les réflexes, les valeurs morales de l'homme moderne. Du point de vue du temps court, historique, c'est la seconde option qui a dominé. Elle a été adoptée en raison de sa capacité à intégrer de larges populations, à s'assurer un avantage militaire sans égal, à développer des potentialités de coopération à grande échelle. Les petites populations anarcho-égalitaires n'étaient pas capables de résister aux formations sociales hiérarchisées en expansion - elles-mêmes généralement hostiles aux premières -, ni de taille à se défendre contre des armées, sauf dans des circonstances exceptionnelles et pour des périodes de temps limitées ${ }^{24}$.

Je postule pour ma part que l'ancienneté et la longue durée d'existence de la modalité anarchique et grégaire (au moins $9 / 10^{\mathrm{e}}$ de sa durée en tant qu'espèce, infiniment plus en tant qu'hominidé) ${ }^{25}$ ont laissé, dans le comportement des êtres humains sociaux hiérarchisés, des traces profondes ${ }^{26}$. Tous membres d'organisations sociales qu'ils soient, tous

[Suite de la note 22] une réalité concrète. Le Trésor public qui dépend de l'État souverain est aussi une caisse commune qui permet la redistribution des richesses. En cela, bien entendu, le capital n'est pas fictif. C'est la construction idéologique de l'État comme être collectif souverain qui l'est. 23. La réalité de l'anti-matière a été prédite par le physicien britannique Paul Dirac en 1931. Il fait l'hypothèse, vérifiée par la suite, de l'existence d'une anti-particule, le positron (cf. l'article «Paul Dirac» de l'encyclopédie libre en ligne Wikipédia).

24. Divers groupes et communautés, au cours de l'histoire, se sont victorieusement opposés aux armées des grands États agraires et même industriels. Cela a été le cas des Cosaques du XVI ${ }^{\mathrm{e}}$ au XVIII ${ }^{e}$ siècle, des communautés de pirates aux XVII et XVIII ${ }^{e}$ siècles et des nations comanche et apache de l'Amérique du Nord au XIX siècle. Sur le temps historique long, ces communautés anarchiques et grégaires ont été défaites et absorbées par les États centralisés qui ont fini par gagner la partie grâce à leur taille démographique et leur puissance militaire.

25. Les ordres de grandeur généralement cités par les préhistoriens et paléoanthropologues sont approximativement de 2,5 millions d'années pour le genre Homo, de 150000 à 200000 ans pour l'espèce Homo sapiens et moins de 10000 ans pour les débuts de l'agriculture et les premiers signes de la révolution néolithique. Que les paramètres de base du comportement humain aient été définis au cours d'une évolution qui a duré des centaines de milliers d'années, voire beaucoup plus, et non pas au cours d'une période de quelques milliers d'années (l'histoire), est une évidence qui tombe sous le sens (cf. Gintis, Van Schaik \& Boehm 2015 : 339).

26. La psychologie évolutionnaire a exploité cette idée de façon sans doute trop littérale avec la théorie des modules neurobiologiques. Même si cette théorie est une sorte de caricature (sous le crâne de l'homme moderne se cache le cerveau d'un chasseur du paléolithique), la présence de structures archaïques chez les êtres vivants est un fait établi. Le passage d'un état à un autre est irréversible et tout état actuel suppose la préservation d'un état antérieur. Ainsi tout être vivant est le fossile d'un être vivant antérieur (Monod 1970). 
hiérarchisés qu'ils deviennent dès l'enfance et tous prisonniers qu'ils restent de la cage corporatiste, des logiques de l'endettement, des habitudes d'obéissance et des fictions transcendantistes, les êtres sociaux ont conservé ces réflexes anciens qui apparaissent si constamment, si régulièrement, si opiniâtrement au sein même de l'ordre social qu'ils dérangent. En d'autres termes, les hommes ont partout une double nature en quelque sorte : anarchogrégaire et sociale, égalitaire et hiérarchique. Certains sont plus grégaires, d'autres plus sociaux, certains sont plus enclins à la liberté, d'autres plus portés à l'autoritarisme, certains sont progressistes, d'autres conservateurs.

La part grégaire et anarchique de l'humanité se manifeste au sein même des ordres sociaux, comme un ferment vivace contraire à l'ordre dans lequel il est enfermé ${ }^{27}$. Nous en avons un exemple sous nos yeux, évident comme la lettre cachée dans la nouvelle d'Edgar Poe. Comment expliquer que les trois mots d'ordre de l'anarchie, "liberté, égalité et fraternité ", soient inscrits au fronton des monuments publics de la République française alors que la société qui construit ces monuments est fondée sur la contrainte étatique, sur l'inégalité des statuts et sur la compétition? Alors que ses institutions supposent nécessairement l'inégalité et la transcendance? Les précédentes considérations expliquent ce fait aisément. La démocratie n'est pas une forme de gouvernement. C'est un processus dans lequel la part anarchique et libertaire tente de rompre l'armature sociale, ou d'en changer radicalement la forme. La démocratie, en ce sens, est une lutte de la société contre elle-même. Ce sont bien d'ailleurs les mêmes individus qui veulent sincèrement l'égalité tout en se soumettant à la hiérarchie, capables de fraternité tout en s'engageant dans la compétition, et aimant les libertés malgré le consentement aux contraintes. Le démocrate veut l'ordre qu'il y a dans le social, mais en même temps il souhaite l'harmonie qui existe dans la grégarité anarchique ${ }^{28}$.

Bien des développements historiques, des phénomènes collectifs, des événements politiques et sociaux, des mouvements populaires, des surgissements existentiels, des systèmes idéologiques peuvent se comprendre

27. La présence d'un mode de vie anarcho-égalitaire se manifeste un peu partout, ne serait-ce que dans les simples réunions entre amis qui créent des espaces de liberté et d'égalité au sein des hiérarchies sociales. Le philosophe et historien anarchiste Peter Lamborn Wilson, écrivant sous le pseudonyme de Hakim Bey, a nommé ce genre d'espaces des «zones d'autonomie temporaire» (Bey 1985).

28. Les historiens et les politologues (par exemple Pierre Rosanvallon [2011]), en cherchant dans le passé récent (inférieur à 10000 ans) l'origine de l'idée d'égalité, ne trouvent évidemment rien d'autre que des discours idéologiques, ceux des philosophies des Lumières ou des philosophes anglais du $\mathrm{XVII}^{e}$ siècle. Ces discours ont construit l'idéologie de l'égalité et de la démocratie telle qu'elle se présente actuellement mais n'expliquent pas le fait anthropologique de l'égalité. 
si l'on tient compte de ces deux sources de la morale (grégaire, anarchique, égalitaire) et de la religion (sociale, hiérarchique), que l'on ne peut pas trouver dans l'histoire, trop courte, mais au contraire dans un passé beaucoup plus lointain.

Cette invention qu'est la société est, à bien des égards, une machine infernale, productrice de guerres dévastatrices, des plus grands massacres à une échelle mondiale, des pires haines, de l'institutionnalisation de la cruauté, des totalitarismes les plus oppressifs. Elle est aussi une boîte miraculeuse d'où l'intelligence magique des hommes fait sortir des merveilles. Y renoncer n'est pas envisageable. Mais si le meilleur de l'homme, son éthique, son altruisme, son goût de la fraternité, de la solidarité et du partage, vient de sa part grégaire, comment envisager l'avenir? Il est possible que celui-ci se joue dans cette dialectique entre l'ordre social et l'harmonie anarchique, dont les termes opposés seront ou non conciliés, amalgamés, réduits ou dépassés.

Centre national de la recherche scientifique, Aix-Marseille Université, Établissement français du sang Unité mixte de recherche Anthropologie bioculturelle, droit, éthique \& santé, Marseille c.macdonald@orange.fr

MOTS CLÉS/KEYWORDS: organisation sociale/social organisation - anarchie/anarchy démocratie/democracy - théorie socio-anthropologique/socio-anthropological theory hiérarchie/hierarchy - égalitélequality - transcendance/transcendence - immanence coopération/cooperation. 
Austin, John L.

1962 How to Do Things with Words.

Oxford, Oxford University Press.

Bey, Hakim

1985 TAZ. The Temporary Autonomous

Zone, Ontological Anarchy, Poetic Terrorism.

New York, Autonomedia.

Boehm, Christopher

1993 "Egalitarian Behavior and Reverse Dominance Hierarchy ", Current Anthropology 34 (3) : 227-254.

1999 Hierarchy in the Forest. The Evolution of Egalitarian Behavior. Cambridge, Harvard University Press.

\section{Brafman, Ori \& Rod A. Beckstrom}

2006 The Starfish and the Spider.

The Unstoppable Power of Leaderless

Organizations. New York, Portfolio.

\section{Buber, Martin}

1992 On Intersubjectivity and Cultural Creativity. Chicago, University of Chicago Press.

Candau, Joël

2012 "Pourquoi coopérer?",

Terrain 58 : 4-25.

\section{Clastres, Pierre}

1974 La Société contre l'État.

Recherches d'anthropologie politique.

Minuit, Paris ("Critique»).

\section{Dentan, Robert K.}

1968 The Semai. A Nonviolent People of Malaya. New York-Chicago, Holt, Rinehart \& Winston ("Case Studies in Cultural Anthropology ").

Diamond, Stanley

1974 In Search of the Primitive.

A Critique of Civilization.

New Brunswick, Transaction Books.
Fiske, Alan Page

1992 «The Four Elementary Forms of Sociality : Framework for a Unified Theory of Social Relations ", Psychological Review 99 (4) : 689-723.

Fox, Robin

2011 The Tribal Imagination.

Civilization and the Savage Mind.

Cambridge, Harvard University Press.

Gardner, Peter M.

2006 Journeys to the Edge.

In the Footsteps of an Anthropologist.

Columbia, University of Missouri Press.

\section{Gibson, Thomas}

1986 Sacrifice and Sharing in the Philippine Highlands. Religion and Society among the Buid of Mindoro. London-Dover, Athlone Press.

Gibson, Thomas \& Kenneth Sillander, eds

2011 Anarchic Solidarity. Autonomy, Equality, and Fellowship in Southeast Asia.

New Haven, Yale University Press («Monograph» 60).

Gintis, Herbert, Carel Van Schaik

\& Christopher Boehm

2015 "Zoon Politikon: The Evolutionary Origins of Human Political Systems ", Current Anthropology 56 (3) : 327-353.

Graeber, David

2007 Possibilities. Essays on Hierarchy, Rebellion, and Desire. Oakland, AK Press.

Granovetter, Mark

1973 "The Strength of Weak Ties", American Journal of Sociology 78 (6) : 1360-1380.

Grauer, Victor

2015 [2011] Sounding the Depths. Tradition and the Voices of History. Kindle version [http ://soundingthedepths.blogspot.fr/] 
Ingold, Tim
1999 "On the Social Relations of HunterGatherer Band ", in Richard Lee \& Richard Daly, eds, The Cambridge Encyclopedia of Hunters and Gatherers. CambridgeNew York, Cambridge University Press : 399-410.

\section{Keil, Charlie}

2010 Born to Groove. Kindle version [http ://borntogroove.org/course/view.php ?i $\mathrm{d}=2$ \& topic=all] .

La Boétie, Étienne de

1995 [1548] Discours de la servitude volontaire. Paris, Mille et une nuits.

Macdonald, Charles J.-H.

1977 Une société simple. Parenté et résidence chez les Palawan (Philippines). Paris, Institut d'ethnologie - Musée de l'Homme ("Muséum national d'histoire naturelle. Mémoires de l'Institut d'ethnologie » 15).

2007 Uncultural Behavior.

An Anthropological Investigation of Suicide in the Southern Philippines. Honolulu, University of Hawai'i Press ("Monographs of the Center for Southeast Asian Studies, Kyoto University English-language Series » 21).

2008a "Order Against Harmony:

Are Humans Always Social ?", Suomen Anthropologi. Journal of the Finnish Anthropological Society 33 (2) : 5-21.

2008b Cooperation, Sharing and Reciprocity. Conférence donnée à la School of Human Evolution and Social Change (University of Arizona). Texte disponible en ligne sur https ://docs.google.com/viewer ?a=v\&pid=s ites\&srcid=ZGVmYXVsdGRvbWFpbnxjaG FybGVzamhtYWNkb25hbGRzc210ZXxne DphNDcyZTcyOTMwYWM2MzM

2009 The Anthropology of Anarchy. Occasional Papers from the School of Social Science 35. Princeton, Institute for Advanced Studies.

2011a "Anarchs and Social Guys ", Society 48 (6) : Symposium Taming the Savage Mind: 489-494. 2011b «Primitive Anarchs: Anarchism and the Anthropological Imagination ", Social Evolution and History 10 (2) : 67-86 [http ://www.sociostudies.org/journal/ articles/140623/].

2011c «A Theoretical Overview of Anarchic Solidarity ", in Thomas Gibson \& Kenneth Sillander, eds, Anarchic Solidarity... : 17-39.

2012a "The Anthropology of Anarchy " Indian Journal of Human Development 6 (1) : 49-66.

2012b "Anthropologia de la Anarquia", Germinal: revista de estudios libertarios 10 : 3-25.

2013 "The Filipino as Libertarian : Contemporary Implications of Anarchism ", Philippine Studies: Historical and Ethnographic Viewpoints 61 (4) : 413-436.

2014a «Joyous, Equal, and Free:

Conditions of Felicity in Human Organizations ", in Michael Egan, ed., The Character of Human Institutions. Robin Fox and the Rise of Biosocial Science.

New Brunswick, Transaction Publishers : 111-131.

2014b «Uncrystallized Society and Libertarian Spirit ", in Masao Nishimura ed., Human Relations and Social Developments. Quezon City, New Day Publishers : 30-49.

Maine, Henry Sumner

1888 [1875] Lectures on the Early History of Institutions. London, John Murray.

\section{Maryanski, Alexandra}

1994 "The Pursuit of Human Nature in Sociobiology and Evolutionary Sociology ", Sociological Perspectives 37 (3) : 375-389.

Maryanski, Alexandra \& Jonathan $\mathrm{H}$. Turner 1992 The Social Cage.

Stanford, Stanford University Press.

Mauss, Marcel

1925 «Essai sur le don : forme et raison de l'échange dans les sociétés archaïques", L’Année sociologique nouv. ser. 1 : 30-86. 
Monod, Jacques

1970 Le Hasard et la nécessité.

Paris, Le Seuil.

\section{Morin, Edgar}

2005 Introduction à la pensée complexe.

Paris, Le Seuil (« Points. Série Essais»).

Ostrom, Elinor

1990 Governing the Commons.

The Evolution of Institutions for Collective

Action. Cambridge-New York, Cambridge

University Press.

Overing, Joanna

2000 "The Efficacy of Laughter",

in Joanna Overing \& Alan Passes, eds,

The Anthropology of Love and Anger.

The Asthetics of Conviviality in Native

Amazonia. London-New York, Routledge : 64-81.

\section{Peterson, Nicolas}

1993 «Demand Sharing: Reciprocity and the Pressure for Generosity Among Foragers ", American Anthropologist 95 (4) : 860-874.

\section{Radcliffe-Brown, Alfred R.}

1951 "The Comparative Method in Social Anthropology ", The Journal of the Royal Anthropological Institute of Great Britain and Ireland 81 (1-2) : 15-22.

\section{Rosanvallon, Pierre}

2011 La Société des égaux. Paris, Le Seuil.

\section{Sade, Donald Stone}

1992 "Dominance Hierarchies as Partial

Orders : A New Look at Old Ideas ", in James Silverberg \& J. Patrick Gray, eds, Aggression and Peacefulness in Humans and Other Primates. New York, Oxford University Press : 57-71.

\section{Sather, Clifford}

1997 The Bajau Laut. Adaptation, History, and Fate in a Maritime Fishing Society of South-Eastern Sabah. Kuala LumpurNew York, Oxford University Press.

\section{Shohet, Merav}

2013 «Everyday Sacrifice and Language

Socialization in Vietnam : The Power
Anthropologist 115 (2) : 203-217.

Stauder, Jack

1972 "Anarchy and Ecology : Political

Society Among the Majangir",

Southwestern Journal of Anthropology 28 (2): 153-168.

\section{Thomas, Elizabeth Marshall}

1989 [1958] The Harmless People.

A Touchstone Book.

New York, Vintage Books.

Tönnies, Ferdinand

1955 Community and Association

(= Gemeinschaft und Gesellschaft).

London, Routledge \& Kegan.

Turnbull, Colin M.

1968 [1961] The Forest People.

New York, Simon \& Schuster.

\section{Turner, Victor}

1974 Dramas, Fields, and Metaphors.

Ithaca, Cornell University Press.

\section{Van Meter, Karl M.}

2014 "Structures of Human Societies", Sociology Mind 4 (1) : 36-44 .

Wenzel, George W., Grete Hovelsrud-Broda

\& Nobuhiro Kishigami, eds

2000 The Social Economy of Sharing.

Resource. Osaka,

National Museum of Ethnology

( Senri Ethnological Studies» $53 »$ ).

West, Stuart A., Ashleigh S. Griffin

\& Andy Gardner

2007 «Evolutionary Explanations

for Cooperation ", Current Biology 17 :

661-672. 
Woodburn, James

1979 "Minimal Politics : The Political

Organization of the Hadza of North

Tanzania ", in William Shack \& Percy S.

Cohen, eds., Politics in Leadership. Oxford,

Clarendon Press.

1982 "Egalitarian Societies ", Man 17 :

431-51.
1998 " "Sharing is Not a Form

of Exchange" : Property-Sharing

in Immediate-Return Hunter-Gatherer

Societies", in Chris M. Hann, ed.,

Property Relations. Cambridge,

Cambridge University Press : 48-63.

RÉSUMÉ/ABSTRACT

Charles Macdonald, Structures des groupes humains: vers une axiomatique. - Cet article propose une théorie unifiée des formes de vie collective. Il en existe deux, reposant sur des principes antithétiques l'une à l'autre : la forme ou modalité anarchogrégaire ou anarcholibertaire qui est ancienne, pré-néolitique, et la forme sociale, socio-hiérarchique, moderne, post-néolithique. Ces deux modalités sont caractérisées, la première par l'équivalence des sujets dans la coopération et par l'égalité, la seconde par la non équivalence des sujets dans la coopération et par la hiérarchie. Ces principes résultent des propriétés essentielles pour les sujets, leurs relations et la structure des groupes. Leur application suppose des mécanismes particuliers à chacune, l'immanence des relations interpersonnelles pour la modalité anarchogrégaire, la transcendance pour la modalité sociale. Ces deux modalités ou formes de vie collective sont présentes ensemble dans toutes les sociétés humaines actuelles et cette dualité fondamentale permet de comprendre des phénomènes idéologiques, sociaux, politiques et historiques. La démocratie est ainsi définie comme une lutte de la société contre elle-même.
Charles Macdonald, Structural Properties of Human Organizations : An Essay in Axiomatics. This essay looks at the possibility of a unified theory of collective organizations. As a starting point the author posits two fundamental variables, autonomy and cooperation. Together they generate two different forms of organizations. One is called "anarchicgregarious" or "weakly-aggregated", the other one is "social-hierarchical». The former has been the rule in pre-Neolithic times and the latter has been dominant in postNeolithic and Historic times. The weakly aggregated form is characterized by complete equivalence between agents, by voluntary cooperation and by maximum individual autonomy. The social-hierarchical option is characterized by the non-equivalent values of agents, by forced cooperation, by hierarchy and reduced autonomy of individual agents. Each requires specific means to operate, the most important being immanence in personto-person relations for the former, and corporate transcendence for the latter. From there on, one can deduct other aspects of collective organizations, such as ideological and religious systems, styles of living, political theories and such. Democracy is tentatively defined as a struggle between two opposite and as yet incompatible principles within extant modern societies. 